\title{
Glycoconjugates on the surface of epididymal spermatozoa in a marsupial, the brushtail possum, Trichosurus vulpecula
}

\author{
N. J. Cooper", R. V. McClean ${ }^{\dagger}$, C. M. Leigh and W. G. Breed ${ }^{\ddagger}$ \\ Department of Anatomical Sciences, Adelaide University, SA 5005, South Australia
}

\begin{abstract}
Variation in localization and distribution of saccharides on the sperm surface of a marsupial, the brushtail possum, Trichosurus vulpecula, was compared between spermatozoa from the caput and cauda epididymides. Spermatozoa were subjected to the following treatments: (i) unfixed and fixed spermatozoa were stained with fluorescein-labelled lectins; (ii) unfixed spermatozoa were incubated with lectins for determination of agglutination; and (iii) spermatozoa were incubated with detergent to remove the plasmalemma, the glycoproteins were separated on SDS-PAGE and western blots were stained with biotinylated lectins. Many of the fluorescein isothiocyanate (FITC)-labelled lectins bound selectively to the sperm surface, and marked differences were found in lectin staining affinity between caput and cauda epididymal spermatozoa. Incubation of spermatozoa from the cauda epididymidis with neuraminidase reversed many of the differences in staining of the cauda epididymal spermatozoa, indicating masking of
\end{abstract}

some terminal saccharides by sialic acid. Agglutination of spermatozoa from the caput epididymidis occurred after incubation with Concanavalin A (ConA) and soybean agglutinin (SBA), but agglutination was less extensive for spermatozoa from the cauda epididymidis. Western blot analysis indicated several ConA-positive bands in caput sperm extracts, but fewer positive bands in the cauda sperm extracts, whereas SBA stained four bands from caput but none from the cauda epididymal spermatozoa. These results demonstrate extensive glycosylation of the surface proteins of spermatozoa from the caput epididymidis and significant differences in spermatozoa from the cauda epididymidis. In general, the findings indicate similar glycosylation of the surface of marsupial spermatozoa to those from eutherian mammals despite marked differences in their morphology and early divergence of marsupials from eutherian mammals. It would appear that this situation differs markedly from that in sub-mammalian vertebrates.

\section{Introduction}

In eutherian mammals, spermatozoa are immature when they leave the testis, but undergo maturation as they travel along the epididymis. During this time spermatozoa undergo marked alterations in molecular composition of the cell surface, which include: (i) a redistribution of the intramembranous protein molecules within the cell membrane; (ii) processing and glycosylation of some of the cell surface proteins and lipids, in part due to the presence of glycosyltransferases within the epididymal environment; and (iii) attachment to the sperm surface of additional molecules synthesized and secreted by the epithelial lining of the epididymis (Bedford, 1975; Orgebin-Crist et al., 1975; Bedford and Cooper, 1978; Jones, 1998; Tulsiani et al., 1998; Schroter et al., 1999, for reviews). The changes in molecular composition of the sperm surface eventually

*Present address: Nerve-Gut Research Laboratory, Department of Gastroenterology, Hepatology and General Medicine, Royal Adelaide Hospital, Adelaide, South Australia

†Present address: School of Animal Studies, University of Queensland, Gatton, Queensland, Australia

${ }^{\ddagger}$ Correspondence

Email: bill.breed@adelaide.edu.au result in sperm maturation such that cauda, but not caput, epididymal spermatozoa have the potential to fertilize an egg.

Marsupials have a considerably different sperm morphology from that of eutherian mammals (Harding, 1987; for a review, see Temple-Smith, 1987; Bedford and Hoskins, 1991) and some of the processes of sperm-egg interaction at fertilization may also differ significantly (Bedford, 1991, 1998). In some of these species, considerable changes in morphology occur in spermatozoa as they travel along the epididymis, including a reorganization of the acrosomal material, shedding of the cytoplasmic droplet and a re-orientation of the sperm head on its axis with the tail (Harding et al., 1979; Harding, 1987; TempleSmith, 1987; Bedford and Hoskins, 1991; Lin and Rodger, 1999). Despite these structural changes, only a few studies to date have investigated the molecular organization of the sperm surface. This situation is surprising as in reptiles and birds, unlike in eutherian mammals, post-testicular sperm maturation appears to involve only minimal glycosylation of the surface proteins (Bedford, 1979; Esponda and Bedford, 1985, 1987). Two studies in marsupials have indicated some differences in the molecular masses of proteins in the cell membrane of cauda compared with caput epididymal spermatozoa (Chaturapanich et al., 1992; 
Lamont et al., 1998), whereas freeze-fracture studies have indicated a considerable change in the arrangement of intramembranous particles of the sperm plasmalemma during epididymal migration in both the New World opossums (Olson, 1980) and the Australian brushtail possum (Cooper et al., 1998). However, apart from a few observations by Temple-Smith and Bedford (1976) and a preliminary study by Cooper et al. (1998), no investigations have been carried out on the surface saccharide composition of spermatozoa of any marsupial, or on the epididymal changes that occur.

Thus, the aim of the present study was to determine the extent of extratesticular glycosylation of sperm proteins in marsupials. Such findings may provide an insight into the uniqueness, or otherwise, of the complex nature of extensive glycoprotein composition of the eutherian sperm plasma membrane and the changes that take place during epididymal maturation.

The distribution of saccharides on the surface of brushtail possum spermatozoa was compared between spermatozoa obtained from the caput and cauda epididymides. Lectins, which have specific binding affinity for particular saccharide groups (for an example, see Goldstein et al., 1997), have demonstrated changes in the distribution of sugars on the surface of eutherian spermatozoa under various physiological conditions (Kinley and Koehler, 1978; for a review, see Koehler, 1978, 1981; Schwartz and Koehler, 1979; Hammerstedt et al., 1982; Margargee et al., 1988; Eddy and O'Brien, 1994). Firstly, fluorescent-labelled lectins were used to determine the distribution and localization of sugars on caput and cauda epididymal spermatozoa of the brushtail possum, Trichosurus vulpecula. Secondly, experiments were conducted to determine whether sperm agglutination occurs after incubation with lectins. Finally, western blots were stained with biotinylated lectins after separation of sperm membrane glycoproteins by electrophoresis.

\section{Materials and Methods}

\section{Animals}

Approximately 20 sexually mature male brushtail possums (Trichosurus vulpecula) were obtained from the Division of Animal Services, Adelaide University. These animals were asphyxiated with carbon dioxide and subsequently injected intracardially with sodium pentobarbital to ensure the animals were dead (Adelaide University Animal Ethics Committee approval S/63/98).

\section{Preparation of sperm suspension}

After the animals were killed, an incision was made in the scrotum, testes and epididymides were removed, and spermatozoa were expressed from the caput and cauda epididymidal ducts. The tissue was placed in Tris-buffered saline, $\mathrm{pH} 7.6$, minced with fine scissors and agitated gently to extrude the spermatozoa, which were aspirated and placed into Eppendorf tubes. Sperm motility was determined by phase-contrast microscopy after placing a drop of the sperm suspension on a glass slide. Only samples with a high percentage of motile spermatozoa were used for subsequent analysis.

\section{Fluorescent labelling of caput and cauda epididymal spermatozoa with lectins}

Caput and cauda epididymal sperm suspensions $(200 \mu \mathrm{l})$ were placed into Eppendorf tubes to which $20 \mu$ l propidium iodide $\left(100 \mu \mathrm{g} \mathrm{m}^{-1}\right.$ in Tris-buffered saline) and $200 \mu \mathrm{l}$ fluorescein isothiocyanate (FITC)-lectin $\left(10 \mu \mathrm{g} \mathrm{ml}^{-1}\right.$ in Trisbuffered saline; Sigma, Cable Hill, NSW) were added, and the solution was incubated for $3 \mathrm{~min}$. The FITC-lectins used included WGA, GSLII, ConA, PSA, RCA, MPL, HPA, DBA, SBA and UEA1 (see Table 1). The sperm-lectin solutions were centrifuged at $300 \boldsymbol{g}$ for 1-2 min, the supernatant was removed and the spermatozoa were resuspended in $200 \mu \mathrm{l}$ Tris-buffered saline. Twenty microlitres of each solution containing the incubated spermatozoa was placed on to a slide, and viewed with an epifluorescent and phase-contrast microscope using emission and absorbance wavelengths for FITC. All experiments were repeated three times, each with material from a different animal, except for those experiments in which spermatozoa were incubated with ConA, which were repeated on material from five different animals. The presence of propidium iodide allowed differentiation of live from dead spermatozoa (Harrison and Vickers, 1990). Only fluorescence of live spermatozoa was recorded. Control experiments were carried out by preincubation of the lectin with the sugar to which the lectin has strong binding affinity (Table 1 ).

In a second series of experiments, spermatozoa were fixed in $2.5 \%(\mathrm{w} / \mathrm{v})$ paraformaldehyde made up in Trisbuffered saline, $\mathrm{pH} 7.6$, for $30 \mathrm{~min}$ at room temperature and subsequently stored at $4^{\circ} \mathrm{C}$. Aliquots $(200 \mu \mathrm{l})$ of fixed spermatozoa were centrifuged at $300 \mathrm{~g}$ for 1-2 min, resuspended in $200 \mu \mathrm{l}$ FITC-lectin solution and treated as described above.

In a third set of experiments, $200 \mu$ l live unfixed cauda epididymal spermatozoa in suspension was incubated in $0.1 \cup$ neuraminidase $\mu \mathrm{l}^{-1}$ for $30 \mathrm{~min}$. The sperm suspension was centrifuged at $300 \mathrm{~g}$ for $5 \mathrm{~min}$ and resuspended in Trisbuffered saline, $\mathrm{pH} 7.6$. FITC-lectin $\left(200 \mu \mathrm{l} ; 10 \mu \mathrm{g} \mathrm{ml}^{-1}\right.$ in Tris-buffered saline) and $10 \mu \mathrm{l}$ propidium iodide $\left(100 \mu \mathrm{g} \mathrm{m} \mathrm{m}^{-1}\right.$ in Tris-buffered saline) were added to each sample and the sample was incubated for $3 \mathrm{~min}$. The sperm-lectin solutions were centrifuged at $300 \mathrm{~g}$ for $5 \mathrm{~min}$ and resuspended in $200 \mu \mathrm{l}$ Tris-buffered saline. Sperm suspension $(20 \mu \mathrm{l})$ from each solution was placed on to a slide, and viewed by epifluorescent and phase-contrast microscopy.

For recording the localization of the fluorescence on the surface of the spermatozoon, the region of the cell membrane was categorized into that over (i) the acrosome, (ii) the rest of the sperm head, (iii) the mid-piece and (iv) the principal piece of the sperm tail. The intensity of staining with FITC-lectins was recorded as weak, moderate or strong 
Table 1. Sugars used to determine specificity of lectin binding to oligosaccharides on the sperm surface of the brushtail possum, Trichosurus vulpecula

\begin{tabular}{ll}
\hline Lectin & Sugar $^{\mathrm{a}}$ \\
\hline WGA $^{\text {b }}$ & $\quad \mathrm{N}$-acetyl- $\beta$-D-glucosamine; sialic acid \\
GSLII & $\alpha$-or $\beta$ - $N$-acetyl-glucosamine \\
ConA & $\alpha$-methyl-mannoside; $\alpha$-methyl-glucoside \\
PSA & $\alpha$-methyl-mannoside; $\alpha$-methyl-glucoside \\
SBA & $\alpha$-and $\beta$ - $N$-acetyl-galactosamine; galactose \\
DBA & $\alpha$ - $N$-acetyl-galactosamine \\
HPA & $\alpha$ - $N$-acetyl-galactosamine \\
PNA & Galactosidyl( $\beta$ - 1,3$)$ - $N$-acetyl-galactosamine \\
RCA 120 & Terminal $\beta$-D-galactose, $\alpha$-galactose, \\
& $\quad N$-acetyl-galactosamine \\
RCA $_{60}$ & $\alpha$-and $\beta$-galactose, $N$-acetyl- $\beta$-D-galactose \\
MPL & $\alpha$-D-galactose, $N$-acetyl-galactosamine \\
UEA1 & $\alpha$-L-fucose
\end{tabular}

ablocking with 0.2 mol sugar $\mathrm{I}^{-1}$ was used in each case for fluorescein

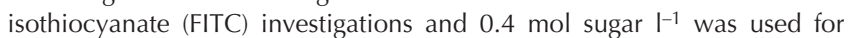
agglutination and western blot analysis.

bSpecificity of WGA for N-acetyl-glucosamine and sialic acid was demonstrated by selective blocking with either $\mathrm{N}$-acetyl-glucosamine or sialic acid.

fluorescence. In unfixed spermatozoa, only the fluorescence of live motile spermatozoa was recorded, which were differentiated from dead spermatozoa by the absence of staining with the propidium iodide. The specificity of lectin binding to particular sugars was determined by prior incubation of the lectin with the relevant sugar (Table 1).

\section{Agglutination studies of caput and cauda epididymal spermatozoa with lectins}

Caput and cauda epididymides were placed into separate dishes containing Tris-buffered saline, $\mathrm{pH} 7.6$, and protease inhibitors. The tissue was minced gently to release spermatozoa into the media. The sperm suspension was placed into a $10 \mathrm{ml}$ tube and centrifuged at $200 \mathrm{~g}$ for $2 \mathrm{~min}$ to remove tissue debris. The supernatant containing the spermatozoa was aspirated and placed into a second tube, which was centrifuged at $500 \mathrm{~g}$ for $10 \mathrm{~min}$ to pellet the spermatozoa. The supernatant was removed and the spermatozoa were resuspended in 1-2 $\mathrm{ml}$ Tris-buffered saline to which protease inhibitors had been added. The number of spermatozoa in the suspension was determined using a haemocytometer, and approximately $1 \times 10^{5}$ spermatozoa were placed into each well of a 24-well tissue culture tray. ConA, PNA, DBA, SBA or UEA1 (Vector Laboratories, Burlington, $\mathrm{CA}$ ) was added at a concentration of $50 \mu \mathrm{g} \mathrm{ml}-1$. Controls included the addition of $0.4 \mathrm{~mol} \mathrm{I}^{-1}$ of the relevant sugar. The sperm-lectin solutions were agitated by rocking for $60 \mathrm{~min}$ in a humid environment at room temperature. The contents of each well were inspected after 60 min to determine the degree of sperm agglutination and were given a score of 0 to 4 as described by Nicolson et al. (1977). For verification of sperm agglutination, an aliquot of spermatozoa from each well was placed on a microscope slide; a coverslip was placed gently on top and the sample was viewed by Nomarski microscopy. From these samples, head-head, head-tail, and tail-tail agglutination were determined. Replicates of spermatozoa from three different animals were carried out.

\section{Determination of molecular masses of glycoproteins by western blot analysis}

Sperm treatment with IGEPAL CA-630 (NP-40) and SDS$P A G E$. Caput and cauda epididymal spermatozoa were squeezed from the epididymis into PBS containing protease inhibitors $\left(300 \mu \mathrm{g}\right.$ EDTA ml-1, $700 \mu \mathrm{g}$ pepstatin $\mathrm{A} \mathrm{ml}^{-1}$, $500 \mu \mathrm{g}$ leupeptin $\mathrm{ml}^{-1}, 1 \mu \mathrm{g}$ aprotinin $\mathrm{ml}^{-1}$ ).

The tissue and spermatozoa in solution were centrifuged at $300 \mathrm{~g}$ for $2 \mathrm{~min}$ to remove tissue debris. The sperm solution was removed, placed into a clean tube and centrifuged at $300 \mathrm{~g}$ for $10 \mathrm{~min}$ to pellet the spermatozoa. The supernatant was removed and the spermatozoa were resuspended in $1 \mathrm{ml}$ PBS plus protease inhibitors. An equal volume of $0.1 \%$ (v/v) IGEPAL CA-630 (NP-40; Sigma) was added to the sperm suspension and the spermatozoa were incubated on ice for $10 \mathrm{~min}$.

After incubation with the detergent, the sperm solution was centrifuged at $500 \mathbf{g}$ for $15 \mathrm{~min}$ to pellet any insoluble components. The supernatant, containing plasmalemma and acrosomal extracts, was removed and placed into a new tube. The protein concentrations were determined using Bradford Protein Assay (BIO-RAD, Hercules, CA), with BSA as standards. The protein samples were concentrated by adding nine volumes of ice-cold acetone and were maintained overnight at $-20^{\circ} \mathrm{C}$. The samples were centrifuged at $500 \mathrm{~g}$ for $20 \mathrm{~min}$ to pellet the proteins, and the supernatant was removed. Samples were washed with five volumes of ice-cold acetone and centrifuged at $500 \mathbf{g}$ for $10 \mathrm{~min}$. The supernatant was removed and the samples were allowed to dry overnight.

Protein samples were solubilized in loading sample buffer under denaturing and reducing conditions.

For SDS-PAGE a ten lane $10 \%(\mathrm{v} / \mathrm{v})$ acrylamide-bisacrylamide separating gel with a $4 \%(\mathrm{v} / \mathrm{v})$ stacking gel was assembled. Samples were boiled for $10 \mathrm{~min}$ and $5 \mu \mathrm{g}$ of each sample was loaded into the appropriate wells. The molecular weights of the bands on the gels were calibrated using commercially available broad range biotinylated standards (BIO-RAD). The gels were run under denaturing and reducing conditions using the buffer system described by Laemmli (1970). Gels were run at a constant current of $45 \mathrm{~mA}$ for $45 \mathrm{~min}$.

Western blot analysis. Protein samples in the polyacrylamide gel were transferred on to nitrocellulose using Towbin's transfer buffer. Transfer was carried out at a constant current of $300 \mathrm{~mA}$ for $50 \mathrm{~min}$. After transfer, the nitrocellulose was stained with $1 \%(\mathrm{w} / \mathrm{v})$ Ponceau $\mathrm{S}$ in $5 \%(\mathrm{v} / \mathrm{v})$ acetic acid which resulted in visualization of the proteins, enabling the 
nitrocellulose to be cut into appropriate strips for subsequent labelling with lectins. Stain was removed from the strips with distilled water and the strips were rinsed in Tris-buffered saline, $\mathrm{pH}$ 7.4. For labelling, the strips were blocked for $2 \mathrm{~h}$ with $3 \%(\mathrm{w} / \mathrm{v}) \mathrm{BSA}$ in Tris-buffered saline, $\mathrm{pH} 7.4$, with gentle rocking and then washed once with Tris-buffered saline for $10 \mathrm{~min}$ to remove excess BSA. The appropriate strips were incubated with either $5 \mu \mathrm{g}$ lectin $\mathrm{ml}^{-1}$ in $0.2 \%(\mathrm{w} / \mathrm{v})$ haemoglobin in Tris-buffered saline or $5 \mu \mathrm{g}$ lectin $\mathrm{ml}^{-1}+0.4 \mathrm{~mol}$ sugar $\mathrm{I}^{-1}$ in $0.2 \%(\mathrm{w} / \mathrm{v})$ haemoglobin in Tris-buffered saline together with a control of $0.2 \%(\mathrm{w} / \mathrm{v})$ haemoglobin in Trisbuffered saline for $1 \mathrm{~h}$, and agitated by gentle rocking. All strips were washed $(2 \times 10 \mathrm{~min})$ with Tris-buffered saline in $0.2 \%(\mathrm{v} / \mathrm{v})$ Tween 20 (TBST). After washing, strips were incubated with streptavidin-alkaline phosphatase (1:3000; Amersham) in TBST for $1 \mathrm{~h}$, with gentle rocking, and washed $(2 \times 10 \mathrm{~min})$ with TBST. The strips were incubated separately for 5-15 min with 5-bromo-4-chloro-indolylphosphate-nitro blue tetrazolium (BCIP-NBT) liquid substrate system, a substrate for alkaline phosphatase, washed with distilled water and left to dry overnight on filter paper.

\section{Results}

\section{WGA and GSLII}

For fresh caput epididymal spermatozoa, FITC-WGA showed bright fluorescence over the acrosome and weak fluorescence over most of the rest of the sperm surface, except for the principal piece of the sperm tail, which did not stain. Prior incubation with $\mathrm{N}$-acetyl-glucosamine reduced, but did not completely block the fluorescence, indicating that much of it was due to $\mathrm{N}$-acetyl-glucosamine, but that some sialic acid may also be present (Table 2). In cauda epididymal spermatozoa, fluorescence was also usually evident over both the head and mid-piece of the sperm tail (Table 2).

Fixed caput epididymal spermatozoa bound FITC-WGA strongly over most of the surface and, in particular, over the sperm tail (Table 3); this fluorescence decreased when $\mathrm{N}$-acetyl-glucosamine was added. Cauda epididymal spermatozoa bound FITC-WGA weakly over the head, acrosome and mid-piece, and the staining was not decreased after prior incubation with $\mathrm{N}$-acetyl-glucosamine, indicating that most of the staining of the cauda epididymal spermatozoa may be due to the presence of sialic acid.

In unfixed caput spermatozoa, GSLII generally only stained the acrosomal region of the sperm head and there was weak staining of the cytoplasmic droplet and midpiece. Cauda epididymal spermatozoa generally did not stain, except occasional weak staining over the mid-piece (Table 2). No staining was observed after prior incubation with $\mathrm{N}$-acetyl-glucosamine, indicating that the fluorescence was due to the presence of $\mathrm{N}$-acetyl-glucosamine.

$R C A_{120}, R C A_{60}$ and MPL

$\mathrm{RCA}_{120}$ usually stained the plasmalemma of caput epididymal spermatozoa over the acrosome and there was weak staining over the mid-piece and the cytoplasmic droplet (Table 2), but no fluorescence was observed after prior incubation with galactose. However, cauda epididymal spermatozoa generally did not stain, except for occasional dull fluorescence over the mid-piece, although prior incubation with neuraminidase resulted in weak fluorescence over the acrosome and mid-piece.

$\mathrm{RCA}_{60}$ showed staining over the head and mid-piece of caput epididymal spermatozoa (Table 2), but staining was not observed after prior incubation with $\alpha$ - or $\beta$-galactose. For cauda epididymal spermatozoa, no binding with $\mathrm{RCA}_{60}$ occurred over any region of the spermatozoon (Table 2), although prior incubation of cauda epididymal spermatozoa with neuraminidase resulted in fluorescence over the sperm head, acrosome and mid-piece, indicating that galactose residues on the surface of caput epididymal spermatozoa had been masked by sialic acid during epididymal transit.

Caput epididymal spermatozoa stained with MPL showed fluorescence over the acrosome and mid-piece (Table 2). In addition, the annulus at the junction between the mid- and principal piece stained brightly. Cauda epididymal spermatozoa generally did not show bright fluorescence over any region, although after incubation with neuraminidase, some fluorescence over both the sperm head and tail was evident, indicating that galactose residues on the sperm surface had been masked by the addition of sialic acid during epididymal transit.

Fixed spermatozoa from the caput epididymidis stained with $\mathrm{RCA}_{120}, \mathrm{RCA}_{60}$ and MPL showed fluorescence over nearly all of the sperm surface, but fluorescence was not observed in spermatozoa from the cauda epididymidis (Table 3).

\section{ConA and PSA}

With either $\alpha$-D-glucose or $\alpha$-D-mannose as inhibitors, it was found that ConA fluorescence was mostly inhibited by prior incubation with methyl mannoside, indicating that ConA may bind largely to the mannose residues.

In caput epididymal spermatozoa, ConA generally stained the acrosomal region of the sperm head, as well as the cytoplasmic droplet and occasionally the mid-piece of the sperm tail. After prior incubation with methyl-mannoside, fluorescence over the acrosome and mid-piece decreased in intensity. For cauda epididymal spermatozoa, there was generally weak fluorescence over the sperm head, and midpiece, and prior incubation with both or either sugar eliminated the fluorescence. Incubations with neuraminidase had no effect on the binding pattern of ConA to cauda epididymal spermatozoa. Binding of FITC-ConA to fixed spermatozoa showed similar results to those obtained for unfixed spermatozoa, except that considerably brighter fluorescence was observed (for a comparison, see Tables 2 and 3).

In unfixed caput epididymal spermatozoa, PSA stained all regions of both the head and tail, and the most intense fluorescence occurred over the acrosome (Table 2). For 
Table 2. Binding of fluorescein isothiocyanate (FITC) lectins to various regions of the plasma membrane of the head and tail of unfixed spermatozoa from the caput and cauda epididymides of the brushtail possum, Trichosurus vulpecula

\begin{tabular}{|c|c|c|c|c|}
\hline & \multicolumn{2}{|c|}{ Plasmalemma of sperm head } & \multicolumn{2}{|c|}{ Plasmalemma over sperm tail } \\
\hline & Acrosome & Rest of head & Mid-piece & Principal piece \\
\hline WGA caput & ++ & + & + & - \\
\hline WGA cauda & + & + & + & - \\
\hline WGA cauda + N & + & + & + & - \\
\hline GSLII caput & + & - & + & - \\
\hline GSLII cauda & - & - & + & - \\
\hline GSLII cauda $+\mathrm{N}$ & - & - & + & - \\
\hline Con A caput & ++ & + & ++ & - \\
\hline Con A cauda & + & + & + & - \\
\hline Con A cauda $+\mathrm{N}$ & + & + & + & - \\
\hline PSA caput & ++ & + & + & + \\
\hline PSA cauda & + & + & ++ & - \\
\hline PSA cauda $+N$ & + & + & ++ & - \\
\hline $\mathrm{RCA}_{120}$ caput & ++ & - & + & - \\
\hline $\mathrm{RCA}_{120}$ cauda & - & - & - & - \\
\hline $\mathrm{RCA}_{120}$ cauda $+\mathrm{N}$ & + & - & + & - \\
\hline RCA $_{60}$ caput & + & + & + & + \\
\hline $\mathrm{RCA}_{60}$ cauda & - & - & - & - \\
\hline $\mathrm{RCA}_{60}$ cauda $+\mathrm{N}$ & + & + & + & - \\
\hline MPL caput & + & - & + & - \\
\hline MPL cauda & - & - & - & - \\
\hline MPL cauda $+\mathrm{N}$ & + & + & + & - \\
\hline HPA caput & +++ & ++ & + & + \\
\hline HPA cauda & ++ & - & - & - \\
\hline HPA cauda $+\mathrm{N}$ & +++ & + & ++ & - \\
\hline DBA caput & + & - & - & - \\
\hline DBA cauda & - & - & - & - \\
\hline DBA cauda $+\mathrm{N}$ & + & - & - & - \\
\hline SBA caput & + & + & + & - \\
\hline SBA cauda & - & - & - & - \\
\hline $\mathrm{SBA}$ cauda $+\mathrm{N}$ & - & - & - & - \\
\hline UEA1 caput & - & - & - & - \\
\hline UEA1 cauda & - & - & - & - \\
\hline
\end{tabular}

Fluorescence was observed with an epifluorescence microscope and scored quantitatively (-: no fluorescence; + : weak fluorescence; ++: moderate fluorescence; +++: strong fluorescence).

Caput: spermatozoa from the caput epididymidis; cauda: spermatozoa from the cauda epididymidis; $\mathrm{N}$ : neuraminidase incubation before staining with lectin.

cauda epididymal spermatozoa, although some variation was evident, fluorescence was generally less apparent, although some fluorescence was observed over the acrosome and mid-piece, but not over the principal piece. When both D-glucose and D-mannose were added, no fluorescence was visible. Neuraminidase had little or no effect on the fluorescence of PSA of cauda epididymal spermatozoa. The binding of FITC-PSA to fixed spermatozoa was generally similar in distribution to that of unfixed spermatozoa, although it was greater in intensity (Table 3). 

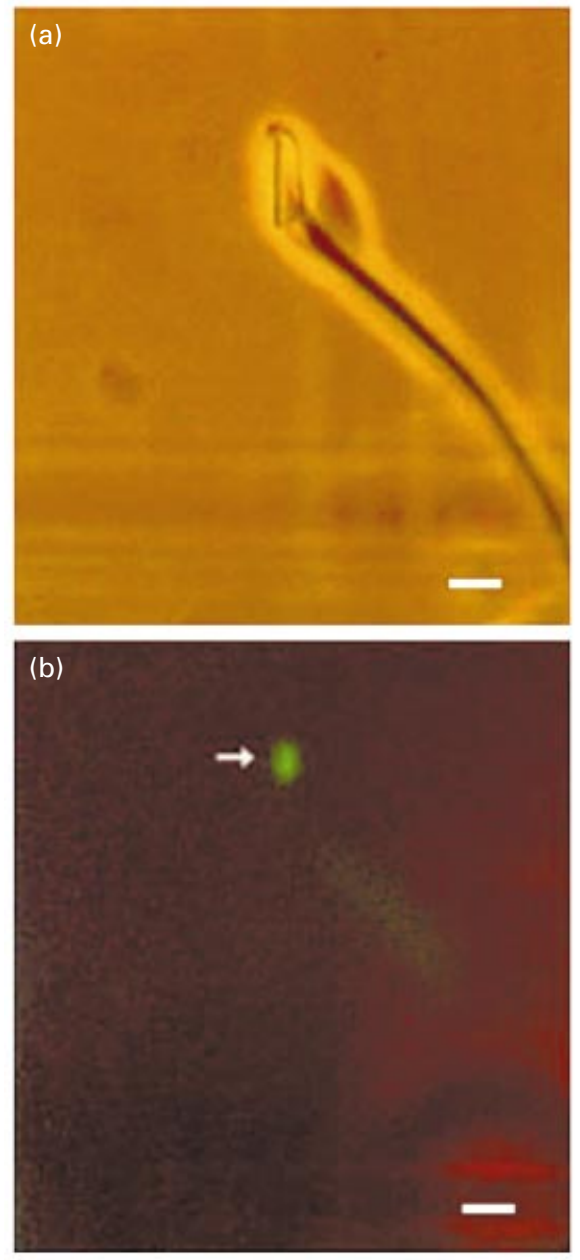

Fig. 1. Matching (a) phase-contrast and (b) fluorescent light micrographs of unfixed spermatozoa from the caput epididymidis of the brushtail possum (Trichosurus vulpecula) showing localization of fluorescein isothiocyanate (FITC)-DBA to the region over the acrosome (arrow). Scale bars represent $2.7 \mu \mathrm{m}$.

\section{$D B A, H P A, S B A$ and UEA 1}

FITC-DBA bound to the plasmalemma of caput epididymal spermatozoa over the acrosome but generally not to other regions of the sperm surface (Table 2; Fig. 1). No fluorescence occurred after prior incubation with this sugar. In some cases the lectin did not bind to any region of cauda epididymal spermatozoa, but in others the region over the acrosome stained weakly. After incubation in neuraminidase, fluorescence was evident over the acrosomal region of the sperm head. The results of fixed spermatozoa were similar to those of unfixed spermatozoa (Table 3).

HPA stained most regions of the caput sperm plasmalemma: intense fluorescence was observed over the acrosome, and moderately intense fluorescence over the rest of the sperm head, cytoplasmic droplet and, to a lesser extent, the mid-piece of the sperm tail (Table 2; Fig. 2). Prior incubation with sugar eliminated this staining. Differences
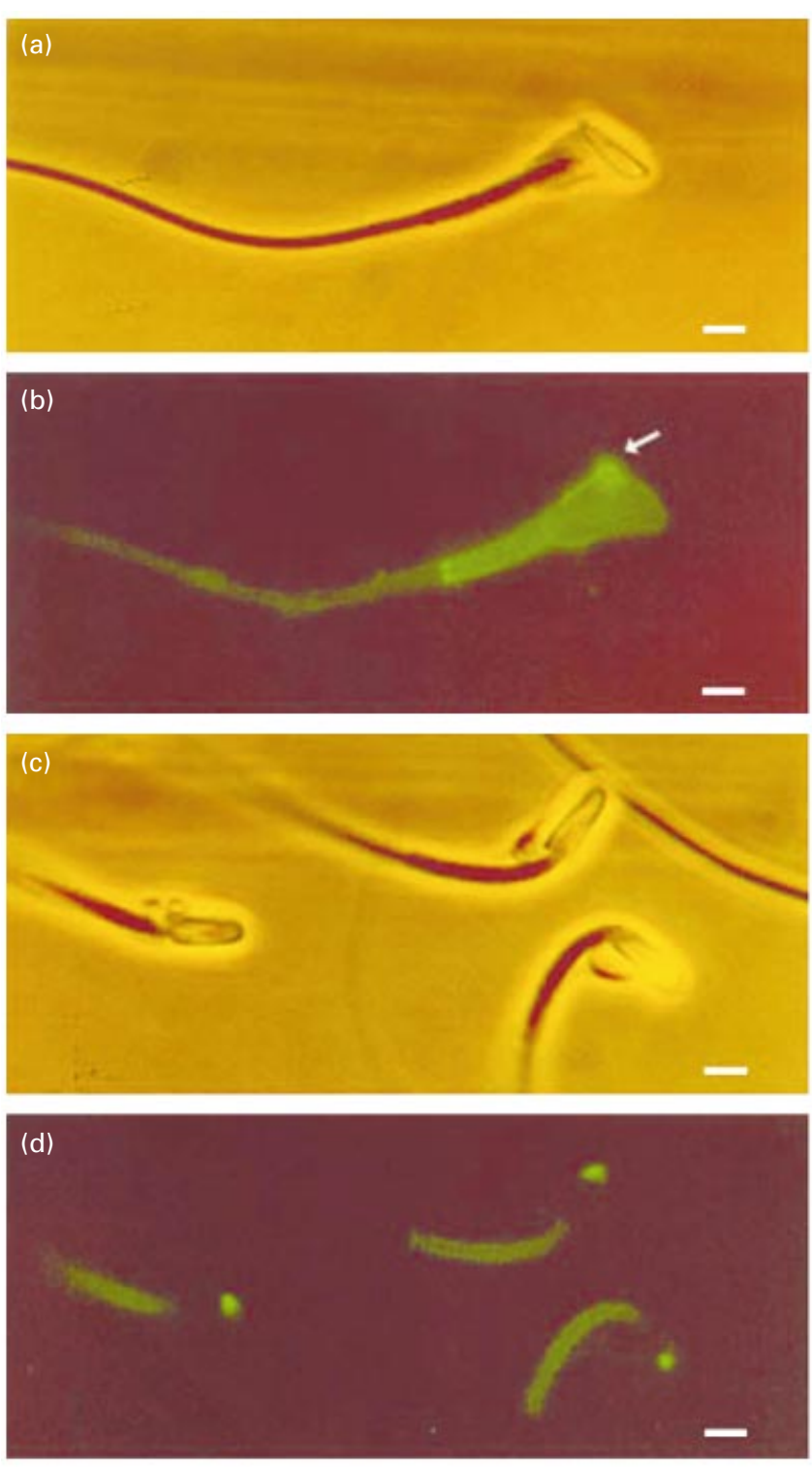

Fig. 2. Matching $(a, c)$ phase-contrast and $(b, d)$ fluorescent light micrographs of unfixed spermatozoa from the caput $(a, b)$ and cauda (c,d) epididymides of the brushtail possum (Trichosurus vulpecula) after staining with fluorescein isothiocyanate (FITC)-HPA. Note that in caput epididymal spermatozoa all of the head and mid-piece fluoresce with lectin, but when the spermatozoa reach the cauda epididymidis, only the regions over the acrosome (arrow) and mid-piece fluoresce. Scale bars represent $2.7 \mu \mathrm{m}$.

in staining were discerned for spermatozoa from the cauda epididymidis, in which staining was observed only over the acrosome, but not over the rest of the sperm head (Table 2; Fig. 2), and the mid-piece showed dull fluorescence in some spermatozoa. After prior incubation with neuraminidase, strong staining over the acrosome and mid-piece was observed, which was blocked by prior incubation with galNAc (Table 2). These results indicate that the decrease in staining of cauda epididymal spermatozoa compared with 
Table 3. Binding of fluorescein isothiocyanate (FITC) lectins to various regions of the plasma membrane of the head and tail of fixed spermatozoa from the caput and cauda epididymides of the brushtail possum, Trichosurus vulpecula

\begin{tabular}{|c|c|c|c|c|}
\hline & \multicolumn{2}{|c|}{ Plasmalemma of sperm head } & \multicolumn{2}{|c|}{ Plasmalemma over sperm tail } \\
\hline & Acrosome & Rest of head & Mid-piece & Principal piece \\
\hline WGA caput & + & + & ++ & ++ \\
\hline WGA cauda & + & + & + & - \\
\hline GSLII caput & + & - & - & - \\
\hline GSLII cauda & - & - & - & - \\
\hline Con A caput & +++ & ++ & +++ & ++ \\
\hline Con A cauda & + & ++ & ++ & - \\
\hline PSA caput & +++ & ++ & ++ & + \\
\hline PSA cauda & ++ & + & ++ & - \\
\hline $\mathrm{RCA}_{120}$ caput & ++ & + & + & + \\
\hline $\mathrm{RCA}_{120}$ cauda & - & - & - & - \\
\hline $\mathrm{RCA}_{60}$ caput & ++ & ++ & ++ & ++ \\
\hline $\mathrm{RCA}_{60}$ cauda & - & - & - & - \\
\hline MPL caput & ++ & + & + & + \\
\hline MPL cauda & - & - & - & - \\
\hline HPA caput & +++ & ++ & ++ & + \\
\hline HPA cauda & + & - & - & - \\
\hline DBA caput & + & - & - & - \\
\hline DBA cauda & - & - & - & - \\
\hline SBA caput & ++ & - & + & - \\
\hline SBA cauda & - & - & - & - \\
\hline
\end{tabular}

Fluorescence was observed with an epifluorescent microscope and scored quantitatively (-: no fluorescence; +: weak fluorescence; ++: moderate fluorescence; +++: strong fluorescence). Caput: spermatozoa from the caput epididymidis; cauda: spermatozoa from the cauda epididymidis.

caput epididymal spermatozoa is due to masking of $\mathrm{N}$ acetyl-galactosamine by sialic acid. Fixed spermatozoa stained with FITC-HPA showed a similar binding pattern to that of the unfixed spermatozoa (Tables 2 and 3).

SBA staining of spermatozoa from the caput epididymidis showed weak fluorescence over the head, mid-piece and cytoplasmic droplet (Table 2), and very weak staining of the principal piece. Fluorescence was eliminated after prior incubation with the sugar. Either weak or no staining of the cauda epididymal spermatozoa occurred, and prior incubation with neuraminidase had no effect on SBA binding. Fixing of the spermatozoa increased the fluorescence over the acrosome and cytoplasmic droplet of caput epididymal spermatozoa, whereas cauda epididymal spermatozoa did not show any fluorescence (Table 3). UEA1 did not result in any fluorescence of either the head or tail of spermatozoa from either the caput or cauda epididymides.

\section{Agglutination of caput and cauda epididymal spermatozoa with lectins}

Incubation of caput epididymal spermatozoa in $50 \mu \mathrm{g}$ ConA ml-1 resulted in agglutination of spermatozoa into large clumps, whereas incubation of the lectin and sperma-

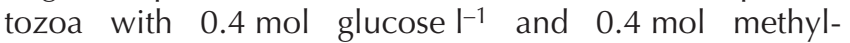
mannoside $\mathrm{I}^{-1}$ inhibited agglutination (Fig. 3). Incubation of spermatozoa from the cauda epididymidis in ConA also resulted in clumping (Fig. 3), but these agglutinated clumps of spermatozoa were not as large as those of caput epididymal spermatozoa. In both caput and cauda epididymal spermatozoa, agglutination was by way of both headhead and head-tail attachment (Table 4), indicating the presence of glucose or mannose over both of these regions of the spermatozoon.

Incubation of caput epididymal spermatozoa with DBA and SBA also resulted in clumping of spermatozoa by way 

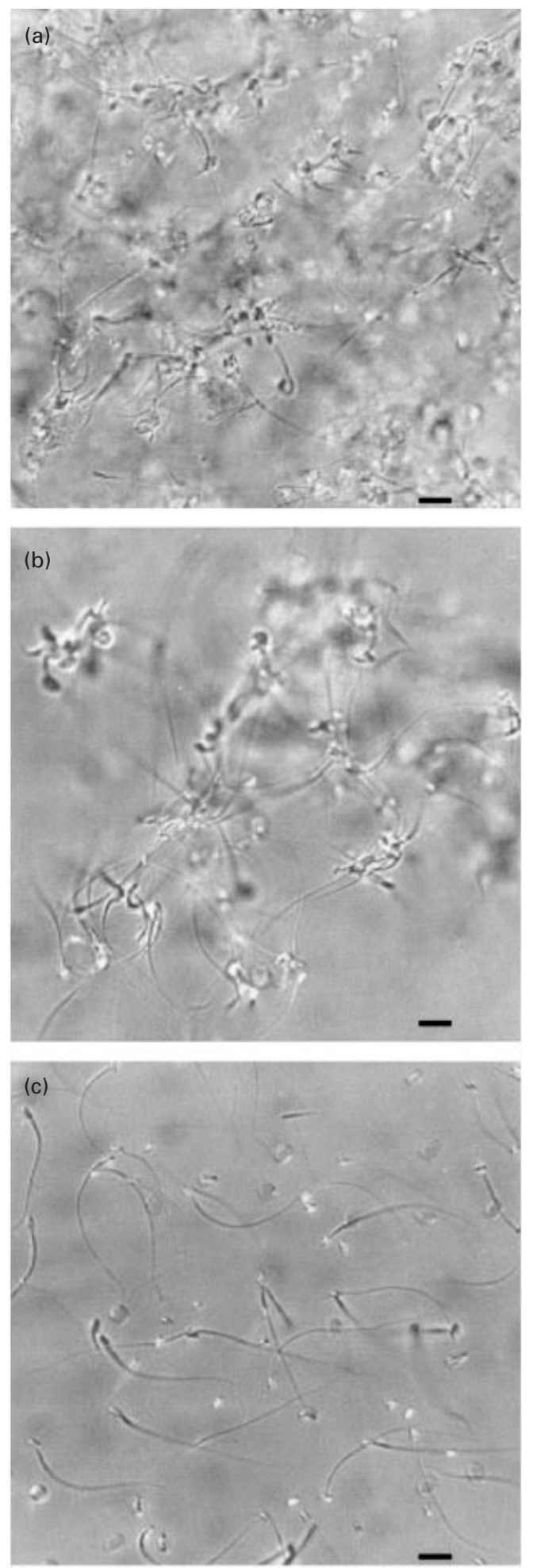

Fig. 3. Nomarski microscopy of brushtail possum (Trichosurus vulpecula) spermatozoa from (a) caput epididymidis and (b) cauda epididymidis after incubation with ConA, and (c) spermatozoa from cauda epididymidis after incubation with glucose and mannose before incubation with ConA. Note clumping of caput and cauda epididymal spermatozoa after incubation in the lectin, but absence of clumping after prior incubation with sugars. Scale bars represent $22 \mu \mathrm{m}$. of head-head or head-tail attachment (Table 4); incubation of spermatozoa and lectin together with the sugar inhibited agglutination. In cauda epididymal spermatozoa, agglutination was less marked and only small clumps occurred by way of head-head interactions; incubation with sugar blocked agglutination.

Incubation of caput epididymal spermatozoa in $50 \mu \mathrm{g}$ PNA $\mathrm{ml}^{-1}$ resulted in clumping of spermatozoa via headtail interactions, whereas incubation of PNA and caput epididymal spermatozoa with $0.4 \mathrm{~mol} \mathrm{~N}$-acetyl-galactosamine $\mathrm{I}^{-1}$ inhibited agglutination. Incubation of cauda epididymal spermatozoa with this lectin did not result in agglutination (Table 4).

Incubation of either caput or cauda epididymal spermatozoa with UEA1 did not result in agglutination.

\section{Determination of molecular masses of glycoproteins stained with WGA and SBA}

Several bands were present in sperm plasma membrane extracts of both caput and cauda epididymal spermatozoa that were stained with biotinylated-ConA, some of which had the same molecular masses as serum proteins. However five bands $(105,86,53,47$ and $39 \mathrm{kDa})$ were present in caput sperm extracts, whereas two bands (>200 and $77 \mathrm{kDa}$ ) occurred in cauda epididymal sperm extracts (Fig. $4 a, b)$ possibly reflecting, in at least two cases, changes in the amount of glycosylation of proteins during epididymal transit. Three bands $(57,55$ and $49 \mathrm{kDa}$ ) were common to both caput and cauda epididymal spermatozoa. Incubation of ConA with 0.4 mol glucose $\mathrm{I}^{-1}$ and 0.4 mol mannose $\mathrm{I}^{-1}$ eliminated binding to all of the observed glycoproteins.

When the sperm extracts were stained with SBA, four bands $(116,103,82$ and $63 \mathrm{kDa})$ were present in caput epididymal sperm plasma membrane extracts (Fig. 4c), whereas no staining was detected in the cauda epididymal sperm plasma membrane extracts. Incubation of SBA with $0.4 \mathrm{~mol} \mathrm{~N}$-acetyl-galactosamine eliminated binding to all observed bands.

\section{Discussion}

In eutherian mammals, numerous studies have demonstrated that, as spermatozoa travel along the epididymis, changes in the distribution and localization of proteins occur within, and on, the plasmalemma. Furthermore, it is also apparent that many of the proteins, and possibly lipids, which are exposed on the cell surface are glycosylated and that the staining for these saccharides changes during epididymal transit (Nicolson et al., 1977; Olson and Danzo, 1981; Hammerstedt et al., 1982; Margargee et al., 1988; Srivastava and Olson, 1991; for a review, see Eddy and O'Brien, 1994). Changes in lectin staining by the time spermatozoa reach the cauda epididymidis indicate a marked alteration in saccharide distribution and abundance during epididymal transit. These changes may be brought about by: (i) the presence of glycosidases and glycosyltransferases within the epididymal fluid; (ii) the secretion 
Table 4. Agglutination of spermatozoa of the brushtail possum, Trichosurus vulpecula, after incubation with lectins

\begin{tabular}{lccccc}
\hline & \multicolumn{2}{c}{ Caput epididymal spermatozoa } & & \multicolumn{2}{c}{ Cauda epididymal spermatozoa } \\
\cline { 2 - 3 } \cline { 5 - 6 } Lectin & Agglutination & $\begin{array}{c}\text { Sperm region showing } \\
\text { agglutination }\end{array}$ & & Agglutination & $\begin{array}{c}\text { Sperm region showing } \\
\text { agglutination }\end{array}$ \\
\hline ConA & 4 (large clumps) & Head-head, head-tail & & 3 (small clumps) & Head-head, head-tail \\
SBA & 4 & Head-head, head-tail & & 2 & \\
DBA & 3 & Head-head, head-tail & & 1 & Head-head \\
PNA & 3 & Head-tail & & 0 & - \\
UEA1 & 0 & - & 0 & - \\
\hline
\end{tabular}

Extent of agglutination of spermatozoa: 0 = no agglutination; 4 = maximum agglutination with no free spermatozoa . Three replicates were carried out.
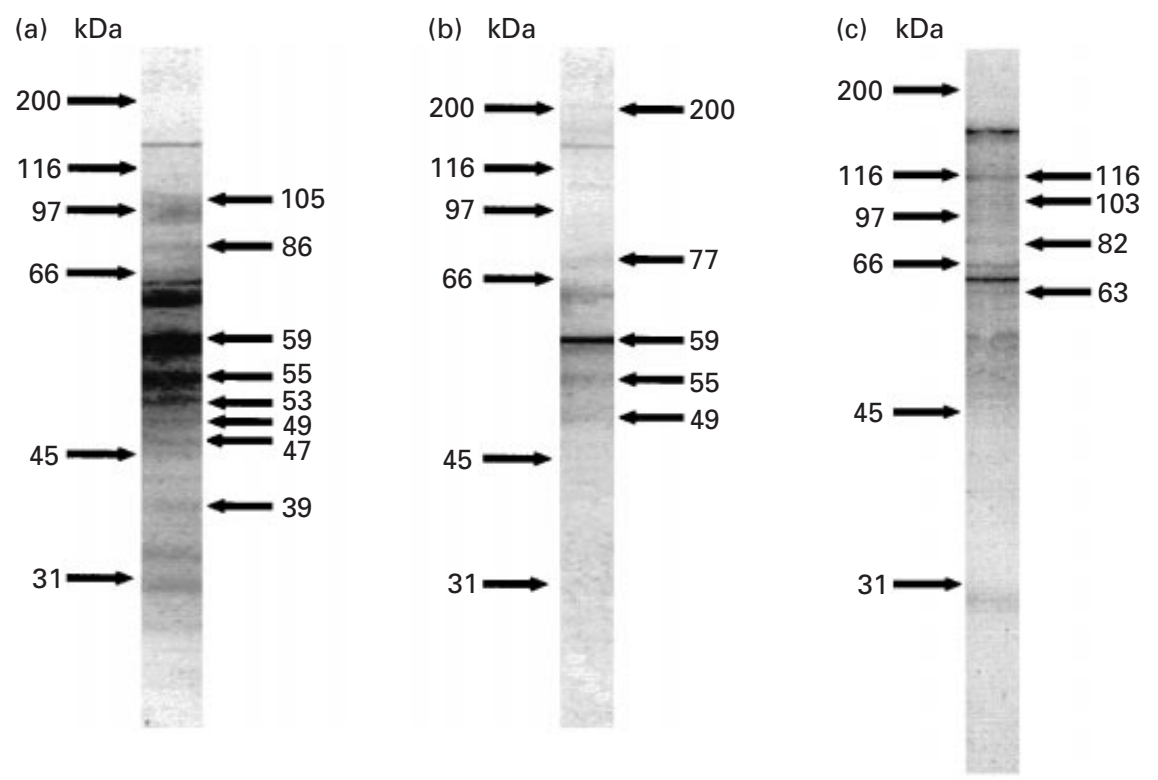

Fig. 4. Western blot analysis of plasma membrane extracts of brushtail possum (Trichosurus vulpecula) (a) caput and (b) cauda epididymal sperm extracts stained with ConA. Note caput epididymal spermatozoa have five bands that were not present in blood plasma $(105,86,53,47$ and $39 \mathrm{kDa})$ and cauda epididymal spermatozoa have two unique bands (> 200 and $77 \mathrm{kDa}$ ) stained with lectin. (c) Caput epididymal sperm extracts stained with SBA showing four bands that were not present in blood plasma $(116,103,82$ and $63 \mathrm{kDa})$. No unique bands were present in cauda epididymal sperm extracts stained with SBA.

of sialic acid by the epididymal epithelium that attaches to the sperm surface and masks the terminal sugar residues; and (iii) loss of saccharides at the time of shedding of the cytoplasmic droplet (Nicolson et al., 1977; Holt, 1980; Margargee et al., 1988). Such changes in the distribution and abundance of surface saccharides may relate, at least in part, to the preparation of the spermatozoon for molecular interactions with the female reproductive tract, capacitation changes and binding of the spermatozoon to the zona pellucida that surrounds the egg.
The present study, which extends the findings of TempleSmith and Bedford (1976) on fixed spermatozoa and the preliminary observations of Cooper et al. (1998), used three approaches to characterize the saccharide content of the possum sperm surface and the changes that take place during epididymal transit. Firstly, caput epididymal spermatozoa stain positively with a variety of fluorescently labelled lectins and show regionalization of staining of some of the lectins. The positive staining on the sperm surface with FITC-WGA and FITC-GSLII indicates $\mathrm{N}$-acetyl-D-gluco- 
samine is present, $\mathrm{RCA}_{120}, \mathrm{RCA}_{60}$ and MPL (together with the effect of the blocking sugars) indicates D-galactose is present; ConA and PSA indicate mannose and $\alpha$-D-glucose are present, whereas DBA, HPA and SBA indicate $N$-acetylgalactosamine is present. The absence of staining with FITC-UEA1 indicates that fucose may not be present on the surface of these spermatozoa. In the fluorescent-labelling studies of unfixed spermatozoa, propidium iodide was used to control for sperm viability (Harrison and Vickers, 1990); thus the staining with FITC-lectins was used to expose saccharide residues on, or over, the plasma membrane of live spermatozoa. The increased intensity of staining that occurred with some of the lectins after fixation was probably due, at least in part, to the lectin-binding of intracellular components as fixation permeabilizes cells.

In the present investigation, when unfixed spermatozoa from the cauda epididymides were stained with the fluorescent-bound lectins, in many cases different results were obtained compared with those obtained with spermatozoa from the caput epididymides. For instance, cauda epididymal spermatozoa unlike caput epididymal spermatozoa did not stain with $\mathrm{RCA}_{120}, \mathrm{RCA}_{60}, \mathrm{MPL}, \mathrm{DBA}$ or SBA, indicating that, similar to the situation in eutherian spermatozoa (Nicolson et al., 1977, Margargee et al., 1988), either loss or masking of various saccharides occurs during epididymal transit. Spermatozoa from the cauda epididymides were also incubated with neuraminidase before the lectin staining to determine which of the two possibilities had resulted in decreased staining. In the case of FITC-RCA 120 , $-\mathrm{RCA}_{60},-\mathrm{MPL}$ and $-\mathrm{DBA}$, but not $-\mathrm{SBA}$, positive staining with the lectin was observed, even though spermatozoa that had not been incubated with the enzyme were negative. This finding indicates that the absence of fluorescence of the cauda epididymal spermatozoa is likely to be due to the secretion of sialic acid and its resultant binding to the sperm surface, and thus the masking of the galactose and possibly some of the $\mathrm{N}$-acetyl-galactosamine residues.

In general, the findings of the agglutination studies support the conclusions from the FITC-labelled lectin observations. Specifically, after incubation with ConA, agglutination was less marked in cauda epididymal spermatozoa than in caput epididymal spermatozoa, indicating that, similar to observations in laboratory rats (Olson and Danzo, 1981), but unlike those observations in rabbits (Nicolson et al., 1977) and rams (Hammerstedt et al., 1982), there were fewer glycoproteins with $\alpha$-D-glucose and $\alpha$-D-mannose on the surface of cauda epididymal spermatozoa. The fact that both head-head and head-tail agglutination occurred strongly supports the results of the FITC-lectin studies, that these sugars are present on the cell membrane of both these regions. Observations from the western blot analysis indicate that, similar to eutherian mammals (Srivastava and Olson, 1991), there are several glycosylated proteins containing glucose or mannose present on the sperm surface of caput epididymal spermatozoa, but by the time the spermatozoa reach the cauda epididymidis there are fewer glycoproteins that stain with ConA.

Results from the western blot analysis with SBA were more marked than those with ConA, as four SBA-positive glycoproteins appeared in caput epididymal sperm extracts, but by the time the spermatozoa reached the cauda epididymides, these differences were no longer apparent. This finding was supported, not only by FITC-labelling studies, but also by the agglutination results indicating that the $\mathrm{N}$-acetyl-galactosamine units to which the SBA binds become reduced during epididymal transit. In relation to these observations, it is possible that exposure of possum spermatozoa to glycosidases, and also to galactosyl transferases, occurs during epididymal transit and that these enzymes cleave some of the glucose and galactosamine residues from the sperm surfaces, as has been suggested in various eutherian mammals (for examples, see Srivastava and Olson, 1991; Srivastava, 2000).

What is the broader significance of the present observations? Even though marsupials diverged from eutherian mammals over 100 million years ago and the morphology of the spermatozoa differ significantly (Bedford, 1991, 1998), it is apparent that the caput epididymal sperm surface has a complex series of surface saccharides similar to that seen in eutherian spermatozoa and that, during epididymal migration, there are modifications in the saccharides on the sperm surface. In eutherian mammals, Bedford (1979) suggested that these changes prepare spermatozoa for capacitation and binding to the zona pellucida. However, in possums, culturing cauda epididymal spermatozoa with oocytes does not result in sperm-zona binding, and recent observations indicate that secretions from the oviduct may be necessary to facilitate binding (Mate et al., 2000). It is possible, therefore, that some of the surface carbohydrates on possum spermatozoa facilitate interaction with the epithelial lining of the female reproductive tract (Suarez, 1998; Topfer-Petersen, 1999) or are involved in protecting the sperm surface molecules during sperm migration (see Schroter et al., 1999). Although possums do not have a distinct series of crypts in the isthmus of the oviduct for sperm storage, unlike those of dasyurid marsupials (Breed et al., 1989; Bedford and Breed, 1994), the spermatozoa may interact with, and may even become bound to, the surface epithelium for a period between mating and ovulation. Thus, this process may involve molecular interaction between saccharides on the sperm surface and those of cells lining the female reproductive tract.

The present study has demonstrated that when the possum spermatozoa enter the epididymis a variety of sugars is already present on their surface and that during epididymal transit a complex series of changes takes place in the saccharide composition. Some of the changes observed in lectin staining may be due to deposition of sialic acid, whereas other changes are probably brought about by the removal of the terminal saccharides by glycosidases and glycosyl transferases in the epididymal 
fluid. The reasons for this complex series of molecular changes in the sperm surface remain to be determined but, in general, the changes appear to be similar to those that take place in spermatozoa of eutherian mammals. In eutherians it has been suggested that the complex series of changes may relate to changes that spermatozoa undergo in the female reproductive tract in preparation for fertilization and, in particular, for those associated with capacitation and the development of sperm-zona binding ability (Bedford, 1979). However, in possums, sperm capacitation is poorly understood and cauda epididymal spermatozoa do not appear to be able to bind to the zona pellucida without secretions from the female reproductive tract. Thus, these changes in carbohydrates on the sperm surface may relate to extratesticular sperm maturation processes other than the development of sperm-zona binding. Alternatively, changes may relate, at least in part, to sperm-female tract interactions that take place between insemination and ovulation.

The authors thank G. Olson for advice and E. Breed for typing the manuscript. This study was made possible by a grant from the Australian Research Council (Grant No. A09802871).

\section{References}

Bedford JM (1975) Maturation, transport and fate of spermatozoa in the epididymis. In Handbook of Physiology Section 7 Endocrinology Vol 5 Male Reproductive System pp 303-317 Eds DW Hamilton and RO Greep. American Physiology Society, Bethesda

Bedford JM (1979) Evolution of the sperm maturation and sperm storage functions of the epididymis. In The Spermatozoon pp 7-21 Eds DW Fawcett and JM Bedford. Urban and Schwarzenberg, Baltimore

Bedford JM (1991) The coevolution of mammalian gametes. In $A$ Comparative Overview of Mammalian Fertilization pp 3-35 Eds BS Dunbar and MG O'Rand. Plenum Press, New York

Bedford JM (1998) Mammalian fertilization misread? Sperm penetration of the eutherian zona pellucida is unlikely to be a lytic event Biology of Reproduction 59 1278-1287

Bedford JM and Breed WG (1994) Regulated storage and subsequent transformation of spermatozoa in the Fallopian tubes of an Australian marsupial Sminthopsis crassicaudata. Biology of Reproduction $\mathbf{5 0}$ 845-854

Bedford JM and Cooper GW (1978) Membrane fusion events in the fertilization of vertebrate eggs. In Cell Surface Reviews, Cell Fusion Vol 5 pp 66-125 Eds G Poste and GL Nicolson. New Holland, Amsterdam

Bedford JM and Hoskins DD (1991) The mammalian spermatozoon: morphology, biochemistry and physiology. In Marshall's Physiology of Reproduction Vol 2 Reproduction in the Male 4th Edn pp 379-568 Ed. GE Lamming. Churchill Livingstone, Edinburgh

Breed WG, Leigh CM and Bennett JH (1989) Sperm morphology and storage in the female reproductive tract of the fat-tailed dunnart Sminthopsis crassicaudata (Marsupialia: Dasyuridae) Gamete Research 23 61-75

Chaturapanich G, Jones RC and Clulow J (1992) Protein synthesis and secretion by the epididymis of the tammar wallaby, Macropus eugenii (Macropodidae: Marsupialia) Reproduction, Fertility and Development 4 533-545

Cooper NJ, Holland MK and Breed WG (1998) Extratesticular sperm maturation in the brushtail possum Trichosurus vulpecula. Journal of Reproduction and Fertility Supplement 53 221-226

Eddy EM and O'Brien DA (1994) The spermatozoon. In Physiology of Reproduction 2nd Edn pp 29-77 Eds E Knobil and JD Neill. Raven Press, New York
Esponda P and Bedford JM (1985) Surface of the rooster spermatozoon changes in passing through the Wolffian duct Journal of Experimental Zoology 234 441-449

Esponda P and Bedford JM (1987) Post-testicular change in the reptile sperm surface with particular reference to the snake, Natrix fasciata. Journal of Experimental Zoology 241 123-132

Goldstein IJ, Winter HC and Poretz RD (1997) Plant lectins: tools for the study of complex carbohydrates. In Glycoproteins II pp 403-455 Eds J Montreuil, JFG Vliegenthart and H Schachter. Elsevier Science, Amsterdam

Hammerstedt RH, Hay SR and Amman RP (1982) Modification of ram sperm membrane during epididymal transit Biology of Reproduction 24 385-392

Harding HR (1987) Interrelationships of the families of the diprotodonta - a view based on spermatozoan ultrastructure. In Possums and Opossums: Studies in Evolution pp 195-216 Ed. M Archer. Surrey Beatty and Sons and the Royal Zoological Society of New South Wales, Sydney

Harding HR, Carrick FN and Shorey CD (1979) Special features of sperm structure and function in marsupials. In The Spermatozoon pp 289-303 Eds DW Fawcett and JM Bedford. Urban and Schwarzenberg, Baltimore

Harrison RAP and Vickers SE (1990) Use of fluorescent probes to assess membrane integrity in mammalian spermatozoa Journal of Reproduction and Fertility 88 343-352

Holt WV (1980) Surface-bound sialic acid on ram and bull spermatozoa: deposition during epididymal transit and stability during washing Biology of Reproduction 23 847-857

Jones $\mathbf{R}$ (1998) Plasma membrane structure and remodelling during sperm maturation in the epididymis Journal of Reproduction and Fertility $\mathbf{5 3}$ 73-84

Kinley WH and Koehler JK (1978) Cell surface changes associated with in vitro capacitation of hamster sperm Journal of Ultrastructural Research $641-13$

Koehler JK (1978) The mammalian sperm surface: studies with specific labelling techniques International Review of Cytology 54 73-107

Koehler JK (1981) Lectins as probes of the spermatozoon surface Archives of Andrology 6 197-217

Lamont AE, Clarke H, Cooper NJ, Holland MK and Breed WG (1998) Protein synthesis and secretion by the epididymis of the brushtail possum, Trichosurus vulpecula. Journal of Reproduction and Fertility 114 169-177

Lin M and Rodger JC (1999) Acrosome formation during sperm transit through the epididymis in two marsupials, the tammar wallaby (Macropus eugenii) and the brushtail possum (Trichosurus vulpecula). Journal of Anatomy $194223-232$

Margargee SF, Kunze E and Hammerstedt RH (1988) Changes in lectinbinding features of ram sperm surfaces associated with epididymal maturation and ejaculation Biology of Reproduction 38 667-685

Mate KE, Sidhu KS, Molinia FE, Glazier AM and Rodger JC (2000) Sperm binding and penetration of the zona pellucida in vitro but not sperm-egg fusion in an Australian marsupial, the brushtail possum (Trichosurus vulpecula) Zygote 8 189-196

Nicolson GL, Usui N, Yanagimachi R, Yanagimachi H and Smith JR (1977) Lectin-binding sites on the plasma membrane of the rabbit spermatozoa Journal of Cell Biology 74 950-962

Olson GE (1980) Changes in intramembranous particle distribution in the plasma membrane of Didelphis virginiana spermatozoa during maturation in the epididymis Anatomical Record 197 471-488

Olson GE and Danzo BJ (1981) Surface changes in rat spermatozoa during epididymal transit Biology of Reproduction 24 431-443

Orgebin-Crist M-C, Danzo BJ and Davies J (1975) Endocrine control of the development and maintenance of sperm fertilizing ability in the epididymis. In Handbook of Physiology Section 7 Endocrinology Vol 5 pp 319-338 Eds DW Hamilton and RO Greep. American Physiological Society, Bethesda

Schroter S, Osterhoff C, McArdle W and Ivell R (1999) The glycocalyx of the sperm surface Human Reproduction Update 5 202-313

Schwartz MA and Koehler JK (1979) Alterations in lectin binding to guineapig spermatozoa accompanying in vitro capacitation and the acrosome reaction Biology of Reproduction 21 1295-1307 
Srivastava A (2000) Maturation-dependent glycoproteins containing both $\mathrm{N}$ - and O-linked oligosaccharides in epididymal sperm plasma membrane of rhesus monkeys (Macaca mulatta). Journal of Reproduction and Fertility $119244-252$

Srivastava A and Olson GE (1991) Glycoprotein changes in the rat sperm plasma membrane during maturation in the epididymis Molecular Reproduction and Development 29 357-364

Suarez SS (1998) The oviduct sperm reservoir in mammals: mechanism of function Biology of Reproduction 51 1105-1107

Temple-Smith PD (1987) Sperm structure and marsupial phylogeny. In Possums and Opossums: Studies in Evolution pp 171-193 Ed. M Archer. Surrey Beatty, Chipping Norton

Temple-Smith PD and Bedford JM (1976) The features of sperm maturation in the epididymis of a marsupial, the brush-tailed possum Trichosurus vulpecula. American Journal of Anatomy 147 471-500

Topfer-Petersen E (1999) Carbohydrate-based interactions on the route of spermatozoon to fertilization Human Reproduction Update 5 314-329

Tulsiani DRP, Orgebin-Crist MC and Skudlarek MD (1998) Role of luminal fluid glycosyltransferases and glycosidases in the modification of rat sperm plasma membrane glycoproteins during epididymal maturation Journal of Reproduction and Fertility Supplement 53 85-97

Received 26 October 2000.

First decision 31 January 2001.

Accepted 12 March 2001. 